\title{
Probiotics in Systemic and Oral Health
}

\section{Saroj M. Bahl*}

Associate Professor, Department of Periodontics, University of Texas-Houston Dental Branch, USA

This article will focus on the potential and established benefits of probiotics for systemic and oral health. Probiotics and prebiotics share a unique role in human nutrition largely centering on manipulation of populations or activities of the bacteria that colonize our bodies. Benefits of regular consumption of probiotics or prebiotics include enhanced immune function, improved colonic integrity, decreased incidence and duration of intestinal infections and reduced incidence of dental caries. The scientific support for health effects and the possible mechanism underlying the action of probiotics is still emerging.

Historically, the concept of probiotics emerged from observations early in the $19^{\text {th }}$ century. Russian Noble prize winner, Elie Metchnikoff hypothesized that Bulgarian peasants owed their health and longevity to the consumption of fermented products. More than 100 years later, it is apparent that microbes have an important influence on immune development and resistance to infections. Microbes are not static colonizers of our bodies but are dynamic symbiotic coresidents. Due to changes in dietary patterns and other environmental alterations, consumption of microbes is much reduced compared to our ancient ancestors. This low exposure is hypothesized to contribute to health problems include the rise in inflammatory and allergic disorders in modern society. The probiotic concept states that consuming the right types of microbes can support the important roles that colonizing microbes play in human health.

According to the Food and Agriculture Organization of the United Nations (FAO) and the World Health Organization (WHO) probiotics are defined as: "live microorganisms, which when administered in adequate amounts, confer a health benefit on the host." To be called a "probiotic", a bacterial strain must be fully characterized. The genus and species of the microorganism must be identified according to internationally accepted methods and its nomenclature corroborated by reference to "Approved Lists of Bacterial Names." Although there is a long list of difference health effects attributable to probiotics, it must be noted that these effects may be associated with specific strains. It cannot be presumed that they all have same effect.

\section{Systemic Benefits of Probiotics}

Potential and established systemic health benefits include:

- Improved intestinal health

- Modulation of the immune response

- Reduced risk of cancer

- Reduced risk of cardiovascular disease

- Improved tolerance of milk [1]

Intestinal conditions that may benefit from consumption of certain probiotic products include diarrhea, Inflammatory Bowel Disease (IBD), Irritable Bowel Syndrome (IBS), Necrotizing Enterocolitis (NE) and Helicobacter Pylori Gastritis. Probiotics may decrease incidence and duration of antibiotic-associated diarrhea. This in turn, may increase adherence to antibiotic therapy such as in opportunistic infections associated with HIV-infection. Inflammatory Bowel Disease (IBD) includes ulcerative colitis and Crohn's disease which are intestinal conditions characterized by a specific relapsing pattern of inflammation of gastrointestinal tract. The origin of IBD is hypothesized to be multifactorial; however, endogenous microbial flora appears to be involved in the etiology. Selected probiotic preparations such as VSL\#3 (consists of 3 Biofido bacterium strains, 4 strains of Lactobacilli and 1 streptococcus strain) prevents relapse of quiescent ulcerative colitis and relapsing pouchitis. However, the beneficial effects last as long as the probiotic treatment is continued. In Crohn's disease among children, a beneficial effect of the tapering of corticosteroids has been described with supplementation of Lactobacillus rhamnosus-GoldinGorbach (LGG).

Another chronic intestinal condition, Irritable Bowel Syndrome (IBS) may benefit from consumption of probiotics. Abdominal pain, flatulence, bloating and diarrhea are associated with this condition and may be difficult to treat. These symptoms may have a significant impact on the quality of the patient's life. After one year of treatment with VSL3, the symptoms were significantly reduced and there was considerable improvement in the gastrointestinal function.

Oral probiotics have been shown to reduce the incidence and severity of necrotizing enterocolitis (NE) in very low birth weight infants [2]. The pathogenesis of NE is multifactorial; major contributing factor appears to be the interaction of bacteria with the premature gut. A low colonization of bifidobateria and lactobacilli in very low birth weight infants in the first month of life has been described. Helicobacter Pylori Gastritis, another intestinal condition has been shown to benefit from treatment with probiotics. Lactobacillus gasseri inhibits growth of the pathogenic bacterium.

In both experimental animals and humans, probiotic cultures have been observed to influence local and systemic immune responses. Various Lactobacillus and Bifidobacteria strains per se or in foods such as yogurt have been demonstrated to enhance nonspecific immunity by enhancing macrophage activation and natural killer cell activity. In human subjects who were consuming a fermented milk product with Lactobacillus acidophilus and Bifidobacteria bifidum, there was no change in lymphocytes but macrophage phagcytosis of $E$. coli increased. Researchers speculate that probiotics have a possible beneficial role in immune response.

Studies conducted in experimental animals indicate that probiotics may reduce the risk of certain cancers such as colon, breast and intestinal cancers. While the data is somewhat contradictory, it is speculated that probiotics may potentially reduce the risk of cardiovascular disease by lowering blood cholesterol levels, increasing resistance of low density

*Corresponding author: Saroj M. Bahl, Associate Professor, Department of Periodontics, University of Texas-Houston Dental Branch, USA, Tel: 713-5004586, E-mail: Saroj.M.Bahl@uth.tmc.edu

Received February 12, 2012; Accepted March 12, 2012; Published March 12 2012

Citation: Bahl SM (2012) Probiotics in Systemic and Oral Health. Dentistry 2:e106 doi:10.4172/2161-1122.1000e106

Copyright: (c) 2012 Bahl SM. This is an open-access article distributed under the terms of the Creative Commons Attribution License, which permits unrestricted use, distribution, and reproduction in any medium, provided the original author and source are credited. 
lipoprotein (LDL) cholesterol to oxidation and reduction of blood pressure.

Alleviation of lactose maldigestion symptoms is well-established for some probiotics and dairy foods containing probiotics. Intake of yogurt made from milk fermented with Lactobacillus bulgaricus and Streptococcus thermophilus has been demonstrated to enhance lactose digestion in individuals with lactase deficiency. This beneficial effect is due to the presence of the enzyme-galactosidase which digests lactose.

\section{Probiotics for Oral Health}

The potential application of probiotics for oral health has recently attracted the attention of several teams of researchers. However, very few clinical studies have been conducted so far. An essential condition for a microbe to represent a probiotic of interest for oral health is its capacity to adhere to and colonize various surfaces of the oral cavity.

Once the bacteria bind to the oral cavity, they secrete various antimicrobial substances, such as organic acids, hydrogen peroxide and bacteriocins. In addition, the microbes compete with the pathogenic agents for adhesion sites on the mucosa. Probiotics can also modify the surrounding environment by modulating the $\mathrm{pH}$ and/or the oxidationreduction potential, which may compromise the ability of pathogens to become established. A combination of probiotics (different strains of bacteria) is often used to increase these beneficial effects.

Clinical studies indicate the usefulness of probiotics in:

- Prevention and treatment of oral infection

- Reduction of dental caries

- Gingivitis

- Periodontal disease

- Halitosis [3]

It is well-recognized that Streptococcus mutans is the most destructive bacterial strain in the mouth as it attaches easily to the teeth and produces a lot of acid. A statistically significant reduction of salivary $S$. mutans was recorded in individuals that consumed probiotic yogurt in contrast to control yogurt. A study conducted in Finland with kindergarten children showed that administration of LGG, as part of milk containing this probiotic, reduced dental decay significantly [4]. Another study demonstrated that Lactobacillus reuteri was efficacious in reducing both gingivitis and dental plaque in patients with moderate to severe gingivitis.

Various studies have reported that the capacity of Lactobacilli to inhibit the growth of periodontal pathogens including Porphyromonas gingivalis, Prevotella intermedia and Aggregatibacter actinomycetemcomitans. A report by Koll-Klais et al. [5] and associates stated that the prevalence of $L$. gasseri and L. fermentum in the oral cavity was greater among healthy participants than among patients with chronic periodontitis. Together, these observations suggest that lactobacilli residing in the oral cavity could play a role in the oral ecological balance.

Halitosis has many causes (including consumption of particular foods, metabolic disorders, respiratory tract infections), but in most cases it is associated with an imbalance of the commensal microflora of the oral cavity. Stated more specifically, halitosis results from the action of anaerobic bacteria that degrade salivary and food proteins to general amino acids, which in turn are transformed into volatile sulphur compounds, including hydrogen sulfide and methanethiol. Kang et al. [6] observed that Weissela cibaria reduced levels of volatile sulfide components by Fusobacterium nucleatum. They concluded that this beneficial effect resulted from the production of hydrogen perioxide by $W$. cibaria which inhibited the proliferation of F. nucleatum. These investigators also found that gargling with a solution containing $W$. cibaria was associated with a net reduction in the production of hydrogen sulfide and methanethiol and consequently a reduction in bad breath.

Probiotics are an emerging area of research in oral health; however, they have been well studied in relationship to systemic/gastrointestinal health. While the preliminary data obtained from several research studies has been encouraging, but numerous randomized clinical investigations are necessary to clearly delineate their relationship to oral health. Studies are also needed for the identification of probiotics that are ideal for oral consumption, as well as the adequate vehicles for their delivery: food products such as cheese, fermented milk, yogurt or supplements such as chewing gum, lozenges etc.

At present, we may not have all the answers; but probiotics certainly represent a promising area of research in oral health for the future.

\section{References}

1. Picard C, Fioramonti A, Francois T, Robinson F, Nenat F, et al. (2005) Review article: Bifidobacteria as probiotic agents-physiological effects and clinical benefits. Ailment Pharmacol Ther 22: 495-512.

2. Douglas LC, Sanders ME (2008) Probiotics and Prebiotics in Dietetic Practice J Am Diet Assoc 108: 510-521.

3. Bonifait L, Chandad F, Grenier D (2009) Probiotics for Oral Health: Myth or Reality?. J Can Dent Assoc 75: 585-590.

4. Näse L, Hatakka K, Savilahti E, Saxelin M, Pönkä A, et al. (2001) Effect of long-term consumption of a probiotic bacterium, Lactobacillus rhamnosus GG, in milk on dental caries and caries risk in children. Caries Res 35: 412-420.

5. Koll-Klais P, Mändar R, Leibut E, Marcotte H, Hammar ström L, et al. (2005) Oral lactobacilli in chronic periodontitis and periodontal health: species composition and antimicrobial activity. Oral Microbiol Immunol 20: 354-361.

6. Kang MS, Kim BG, Chung J, Lee HC, Oh JS (2006) Inhibitory effect of Weissella cibaria isolates on the production of volatile sulphur compounds. $J$ Clin Periodontol 33: 226-232. 